\title{
Impact of non-verbal communication on collaboration in 3D virtual worlds: case study research in learning of aircraft maintenance practices
}

\author{
Gustavo Silva ${ }^{1}$, Leonel Morgado ${ }^{1,2}$, Armando Cruz $^{3}$ \\ ${ }^{1}$ Universidade Aberta, Lisbon, Portugal \\ 2 INESC TEC, Porto, Portugal \\ ${ }^{3}$ Instituto Politécnico de Viseu, Viseu, Portugal \\ gjmsilva@outlook.com, Leonel.Morgadoduab.pt, \\ cruz.armandolesapo.pt
}

\begin{abstract}
Virtual worlds are widely regarded as a successful approach for professional team training, due to promoting collaboration through user avatars. Current theoretical models of Computer-Supported Cooperative Work (CSCW) do not account for the specificity of virtual worlds' affordances for collaboration, particularly regarding non-verbal communication affordances, which led Cruz et al. to propose in iLRN in 2015 a specific protocol to collect data to support the empirical-grounded refinement of the theoretical treatment of virtual worlds in CSCW. Here, we present the results of applying this protocol to a case study in the training of aircraft maintenance practices. A training simulator platform was built with the Open Simulator platform for this purpose. Through activity observation, collected data provided a set of chains of evidence as proof elements, increasing the amount of empirical data available for future theory development.
\end{abstract}

Keywords: virtual environments, virtual worlds, collaboration, presence, nonverbal communication, aircraft maintenance.

\section{Introduction}

Three-dimensional Virtual Worlds (3DVW) offer users the opportunity to feel immersed in a digital environment that simulates the physical environment [1] and create immersive environments conducive to collaboration among users. Collaboration can be analysed with the theoretical framework of Computer Supported Cooperative Work (CSCW) [2] but its taxonomies fail to address the key characteristics of 3DVW [3]. Cruz et al. sustained that the impact of non-verbal communication on collaboration in 3DVW is not well known, even considering that some characteristics, such as embodiment in an avatar and immersion within a (virtual) physical space, support this type of communication [4]. It has thus been called forward the necessity to have more empirical data about this problem to increase the degree of confidence in data and possibly develop CSCW theory frameworks [3]. Thus, our approach presented herein

adfa, p. 1, 2011.

(C) Springer-Verlag Berlin Heidelberg 2011 
was the development of a case study focused on training in aeronautical maintenance practices, to collect new data about the impact of non-verbal communication in a cooperative learning context. The case study was carried out at CENFORTEC, a training centre for aircraft maintenance technicians (AMT), located at the Cascais (Portugal) municipal airfield. With this work, we provide new data, collected using the protocol proposed by Cruz et al. in iLRN 2015 [3] and subsequently detailed in 2016 [4], but with a new case study that can contribute to improve the knowledge about the theoretical model of CSCW. The data was extracted from an aeronautical practices scenario. We related the evidences obtained with the propositions defined by Cruz et al. [4]. The analysis of the data identifies several chains of evidences, replicating some of them found in the scenario analysed by Cruz et al., and showed new chains of evidences.

\section{Background}

3DVW are collaborative virtual environments allowing users to feel immersed inside them, through a graphic representation called avatar, which can communicate with other users' avatars using audio, video, and gestures, among other possibilities [6]. CSCW is an interdisciplinary scientific area that studies how information technologies can aid group work [12]. It is an area that has driven the application and systematic evaluation of avatar interaction and features because they have desirable effects on the experience of social presence on the sense of task execution [10].

The sense of presence, in particular is important to understand collaboration in 3 DVW [5]: it has been shown that these worlds enable users to interact in a way that contributes to the sense of presence [7]. Collaboration itself relates to the sense of presence [9] and it is also recognized that immersion, non-verbal information [8], communication [10] and interaction [5;11] are important to the sense of presence. Non-verbal communication can be used to improve awareness, the sense of belonging to a group, and collaborative tasks in general [5], [10].

In general, there is a broad consensus on the importance of the communication functions of non-verbal communication, but there is still little knowledge about its specific mechanisms [8]. In particular, there is a lack of data about the relevance of low-level subtle and transient effects inherent to non-verbal communication, like the use of specific gestures and coordination of verbal and non-verbal activities [10]. This is a limitation of the ability of current CSCW theoretical frameworks to handle 3DVW [13] that this work aims to address by collecting evidences towards building up data that may eventually enable reshaping those frameworks.

\section{Methodology overview}

This work adopted the case study research methodology following Yin's perspective which sees it as "an empirical investigation that investigates a contemporary phenomenon within its real-life context, especially when the boundaries with the context are not clearly defined, or the investigator has little control over these points" [14]. 
This methodology comprises 1) Research Design, 2) Preparation for Evidence Collection, 3) Evidence Collection, 4) Evidence Analysis and 5) Sharing of Results. Cruz et al. defined the first two steps for our specific problem as part of their case study protocol [4]. The Research Design contains: Research questions that provide an important key, establishing the most relevant research strategy to be used; Propositions, which direct attention to what should be examined within the study objective; units of analysis, which relate the data with the fundamental problem, linking them to propositions; and finally, the definition of a criterion for the interpretation of the results obtained [14], [15]. We applied this protocol to a scenario developed in OpenSimulator, using its research questions (as well as its propositions and units of analysis):

RQ1: How does the use of an avatar influences collaboration 3DVW?

RQ2: How does the virtual spatial environment influences collaboration 3DVW?

Our case study consists of a simulation in a 3DVW (built using OpenSimulator) of a learning activity on the process of aircraft towing. The participants, within the scope of a training-learning activity, collaborate in the required tasks. Data about interactions between the participants and the environment were collected by direct observation.

\section{$4 \quad 3 D V W$ aircraft towing simulator}

Aircraft maintenance is a complex, rigorous, delicate and exhaustive process that requires a very precise training program [16]. Therefore, is essential to undergo proper maintenance training to ensure that aviation maintenance technicians have the qualification, proficiency, and certification required to perform their tasks [17]. It is in this context that simulation in 3DVW arises as an alternative way to obtain part of this training by providing new opportunities that can be used by trainers and trainees [18].

We developed an e-learning module for this purpose and provided it on a Moodle e-learning software platform at the CENFORTEC (Cascais, Portugal) training centre for aircraft maintenance technicians. This e-learning module allowed a group of trainees to acquire knowledge on how to conduct a maintenance activity, aircraft towing, in a simulation scenario. The module is organized into topics: 1) Introduction, describing the objective of aircraft towing training; 2) Security issues that users must respect; 3) Constitution of the tow crew and responsibilities of the participants; 4) Description of the aircraft towing process; 5) Equipment used in the towing process; 6) Signals defined by International Civil Aviation Organization (ICAO) for nonverbal communication; 7) Introduction to the simulation environment. It also included a tutorial for installation and setup of the 3DVW viewer to access the scenario.

The simulator was built specifically to support this theoretical module, using Open Simulator for the server, and made accessible through the Internet. Users accessed the virtual environment through the Firestorm client viewer.

The simulator scenario intent was to be visually simple but sufficiently credible for the trainees, who are familiar with the aeronautical environment. It was constructed from scratch, modelling all necessary objects with the tools made available by Firestorm. The 3D graphical modelling of the scenario recreated an airfield (Figure 1): a 
runway, oriented $10^{\circ}$ north, $190^{\circ}$ south, a main apron and a taxiway parallel to the runway, linking the runway to the main apron. The set "tractor - tow bar - aircraft" parked on the taxiway is used in the simulation, and the remaining aircrafts are only for decorative purposes, with the intent of supporting the overall context. The scenario also has some user-support areas: billboards with topics on towing procedures, a training area for towing tractor practice, and an area with a video on towing procedures. The specific movements/gestures defined by ICAO were built with third-party software and loaded into the viewer for use by avatars. The name above the head of each avatar, combined with his/her uniform identified that avatar and the role he/she played. Trainee avatars wore a yellow shirt, light blue coat (optional) and black trousers, and the trainer avatar wore a white shirt with dark blue trousers. Each trainee had a specific task within the training setting.

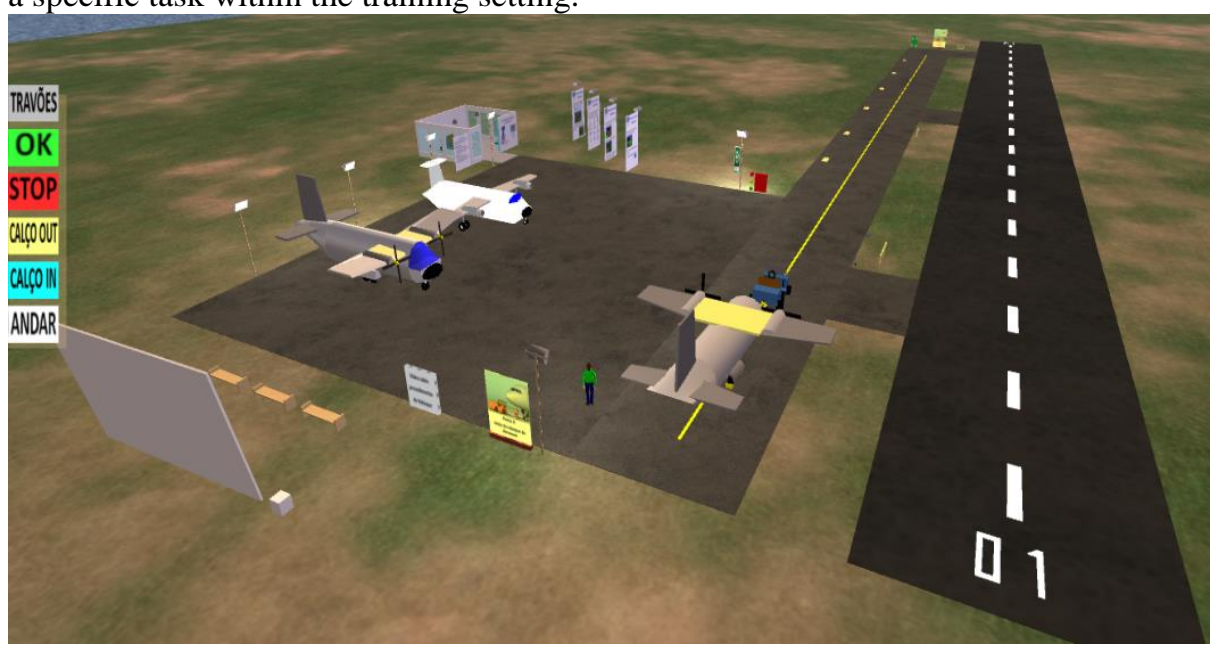

Figure 1 - The simulator scenario, with the aircraft to be towed and the airstrip.

\section{$5 \quad$ Evidence Collection}

Six avatars took part in the simulation (a trainer, a cameraman, and four trainees). At the beginning of the session, the trainer appointed a trainee as crew chief, who in turn, appointed the other crew elements (a tractor operator and two wing observers). Afterwards, they went to the aircraft to perform the aircraft towing procedures.

The interactions between avatars, and of avatars with objects in the simulator, enabled the collection of data. The observation of the evidences was made directly from the case and followed the process used by Cruz et al. [13]: "each reference to the use of a feature or behaviour was accounted for, relating to one or more units of analysis, according the impact of the evidence described in the unit". Collected data were organized for the case study in a two-table database [15]: 
Table 1. Evidences of the units of analysis related to the avatar

\begin{tabular}{|c|c|}
\hline Units of Analysis & Evidences \\
\hline Appearance & $\begin{array}{l}\text { All avatars were dressed with uniforms. } \\
\text { All trainee avatars wore the same uniform, but a different one from } \\
\text { the trainer. } \\
\text { The trainees positioned themselves close to each other and turned } \\
\text { to face the trainer as he gave explanations about the session. }\end{array}$ \\
\hline Gestures made & $\begin{array}{l}\text { The avatars performed several gestures during the session. } \\
\text { Beams of coloured balls were seen leaving some avatars' hands } \\
\text { towards objects, and subsequent changes in their responses. } \\
\text { Circular beads of coloured balls were seen next to the objects with } \\
\text { which the avatar was interacting. } \\
\text { Some avatars made gestures depicting writing on a keyboard, } \\
\text { indicating to others that they were communicating by text using } \\
\text { the instant messaging (IM) avatar-to-avatar communication sys- } \\
\text { tem. } \\
\text { After the crew chief made the sign "chocks out", beams came out } \\
\text { of the hands of some avatars towards the main landing gear. } \\
\text { At the end of the tow, after the crew chief made the sign "chocks } \\
\text { in", beams came out of the hands of some avatars towards the } \\
\text { main landing gear. } \\
\text { The avatars used various gestures defined by ICAO to communi- } \\
\text { cate with each other without using audio or IM channel. Gestures } \\
\text { were used to pass the following messages: "chocks out", "chocks } \\
\text { in", "stop", "go ahead", "ok/free". }\end{array}$ \\
\hline Observation & $\begin{array}{l}\text { After an avatar raised his arm and waved with his hand, the trainer } \\
\text { interrupted his activities, turned to him, and asked him to present } \\
\text { his doubts. } \\
\text { After the observer avatars made the gesture "ok", upon finishing } \\
\text { the airplane inspection, the avatar crew chief also made the gesture } \\
\text { "ok", returning the communication. } \\
\text { During the tow, the crew chief saw an obstacle and made the ges- } \\
\text { tures to signal that the crew should stop the aircraft towing. }\end{array}$ \\
\hline $\begin{array}{l}\text { Direction of } \\
\text { movement }\end{array}$ & $\begin{array}{l}\text { At the beginning of the training session the trainer addressed the } \\
\text { trainees who gathered close to each other near the meeting point. } \\
\text { After being named, each avatar went to the place previously de- } \\
\text { fined in the theoretical content (crew chief to the front of the trac- } \\
\text { tor, tractor operator next to the crew chief, and observers near the } \\
\text { wing tips of the aircraft). } \\
\text { After an order of the crew chief, the avatar operator sat on the } \\
\text { tractor. } \\
\text { At the beginning of the tow the avatar crew chief made the gesture } \\
\text { corresponding to the tractor moving forward, and the operator set } \\
\text { the tractor in motion towards the crew chief. }\end{array}$ \\
\hline
\end{tabular}


Avatar placement At the beginning and at the end of the session, the avatars trainees gathered in front of the trainer avatar to listen to their explanations. The avatars moved within the scenario and positioned near the objects (tractor, aircraft, etc.) with which they interacted.

Table 2. Evidences of the units of analysis related to the physical space

\begin{tabular}{ll}
\hline Units of analysis & Evidences \\
\hline Animated visual & The set "tractor - tow bar - aircraft", through interaction of the \\
artifacts & $\begin{array}{l}\text { avatar, moved forward / backward, etc. } \\
\text { In the support area, near the meeting point, there was an instruc- } \\
\text { tion panel and a tractor that allowed training of the use of the trac- } \\
\text { tor by the avatar. }\end{array}$ \\
& The cockpit, the nose landing gear, and the main landing gear, \\
Non-animated & visually changed (or displayed messages) when an avatar interact- \\
visual artifacts & ed with them. \\
& A coloured panel at the beginning of the taxiway, indicated wheth- \\
& er it could be used (green panel) or if it was interdicted (red panel). \\
& All avatars presented their names above their heads. \\
& The interactions by avatars caused some scripts to run and execute \\
Non-visual arti- & actions in response to those interactions. \\
facts & The session was scheduled by an e-mail sent to trainees. \\
& Avatars communicated through Google Hangouts audio group and \\
& sometimes avatars used OpenSimulator text IM. \\
& The session took place in a space simulating part of the area of an \\
airfield: a main apron containing aircrafts, a runway, and a taxiway & where the aircraft tow took place. In the taxiway is a set "tractor, \\
Visual environ- & tow bar, aircraft" to be towed from point "A" to point "B". \\
ment &
\end{tabular}

\section{Evidence Analysis}

The analysis of results was accomplished according to the general analytical strategy defined by Yin [14]. That is, linking the collected data to the stated propositions, thus creating one or several chains of evidence for each proposition. To accomplish this, table 3 was constructed with the chain or chains of evidence that support each proposition defined by Cruz et al. (evidences, related evidences, additional evidences) [4]. A chain of evidence starts with a reference to a unit of analysis extracted from the case, and in the other columns are provided evidences of behaviors that are suspected to be caused by that reference to the unit of analysis, or by a behavior provoked by that reference. Thus, a chain of behaviors is created. Those evidences are also extracted from the case. In this table, each proposition is related to the chains of evidences that support them, by assigning the chains to the propositions if the reference to the unit of analysis in the beginning of each, is itself, related to the proposition in cause. The chains of evidence marked with "*” are new, the others match those already iden- 
tified by Cruz et al. The new chains bring new data about non-verbal communication in 3DVW.

Table 3. Propositions and related evidences/units of analysis

P1: The aesthetics of the avatar influence the perception by others of the role of the avatar and/or his attitude

All avatars were dressed All trainee avatars wore the The theoretical module prowith uniforms. same uniform, but a different one from the trainer vided in Moodle showed the functions and activities that the trainees should perform coordinated by the trainer.

P2: The gestures and sounds that the avatar does, influence the perception by others about how the avatar's user wants to collaborate or how he/she wants others to collaborate.

Avatars made several At the beginning and end of the The users communicated gestures during the session, the trainees' avatars through Google Hangouts session. gathered in front of the trainer audio group, and sometimes avatar to listen to their explana- avatars used IM, communitions. cating via text.

P3: The eye gaze/face direction, direction of movement, and avatar placement provide cues about what the user is playing attention to, or what the user would like to direct others' attention to.

\begin{tabular}{lll}
\hline $\begin{array}{l}\text { The avatars moved } \\
\text { within the scenario and } \\
\text { positioned themselves } \\
\text { near the objects (tractor, } \\
\text { aircraft, etc.) with } \\
\text { which they interacted.* }\end{array}$ & $\begin{array}{l}\text { Beams of coloured balls were } \\
\text { seen leaving avatars' hands } \\
\text { towards objects and subsequent } \\
\text { changes in position or move- } \\
\text { ment. }\end{array}$ & $\begin{array}{l}\text { After the crew chief made the } \\
\text { sign "chocks out", there were } \\
\text { hands of some avatars to- } \\
\text { wards the main landing gear.* }\end{array}$ \\
\hline $\begin{array}{l}\text { Avatars used ICAO } \\
\text { gestures to communi- } \\
\text { cate without using audio } \\
\text { or IM text channel.* }\end{array}$ & $\begin{array}{l}\text { Avatars performed several } \\
\text { gestures during the session } \\
\text { (sending messages; communi- }\end{array}$ \\
\hline
\end{tabular}

P4: Interaction of the avatar with specific objects provides cues about which objects are intended to be used by others in the collaboration process.

\begin{tabular}{|c|c|c|}
\hline $\begin{array}{l}\text { After an order of the } \\
\text { crew chief, the avatar } \\
\text { operator sat on the } \\
\text { tractor.* }\end{array}$ & $\begin{array}{l}\text { Crew chief made the gesture } \\
\text { corresponding to the tractor } \\
\text { moving forward, and the opera- } \\
\text { tor set the tractor in motion } \\
\text { towards the crew chief.* }\end{array}$ & $\begin{array}{l}\text { The set "tractor - tow bar- } \\
\text { aircraft", through interaction } \\
\text { of the avatar operator, showed } \\
\text { movement forward / back- } \\
\text { wards, etc.* }\end{array}$ \\
\hline \multicolumn{3}{|c|}{$\begin{array}{l}\text { P5: The arrangement of objects (e.g. their grouping or alignment) provides cues of their } \\
\text { purpose for collaboration. }\end{array}$} \\
\hline $\begin{array}{l}\text { The set "tractor - tow } \\
\text { bar- aircraft", moved } \\
\text { forward / back, etc.* }\end{array}$ & $\begin{array}{l}\text { Cockpit and nose \& main land- } \\
\text { ing gears visually changed (or } \\
\text { displayed messages) when an } \\
\text { avatar interacted with them.* }\end{array}$ & $\begin{array}{l}\text { The interactions by avatars } \\
\text { caused some scripts to run } \\
\text { and execute actions in re- } \\
\text { sponse to these interactions.* }\end{array}$ \\
\hline
\end{tabular}




\begin{tabular}{lll}
\hline $\begin{array}{l}\text { A coloured panel at the } \\
\text { beginning of the taxi- } \\
\text { way indicated whether }\end{array}$ & $\begin{array}{l}\text { Circular beads of coloured balls } \\
\text { were seen next to the objects in } \\
\text { which the avatar was interact- }\end{array}$ & $\begin{array}{l}\text { At the beginning of the tow, } \\
\text { the avatar crew chief made } \\
\text { the gesture corresponding to }\end{array}$ \\
$\begin{array}{l}\text { ing: The panel turned green } \text { or if it was inter- } \\
\text { ane thactor moving forward, } \\
\text { ticted (red panel).* }\end{array}$ & $\begin{array}{l}\text { afterainer interaction.* } \\
\text { and the operator set the trac- } \\
\text { tor in motion towards the } \\
\text { crew chief.* }\end{array}$ \\
\hline
\end{tabular}

P6: The exchange of visual artifacts (i.e., "objects", "clothes", "tools"), with the specific visual features and explicit purposes, helps define the team, contributing to group awareness, and perception of collaboration roles.

\begin{tabular}{ll}
\hline $\begin{array}{l}\text { All the avatars showed } \\
\text { their name hovering } \\
\text { over their heads. }\end{array}$ & $\begin{array}{l}\text { The trainee avatars positioned } \\
\text { themselves close to each other chef, and the crew chief } \\
\text { and turned to the trainer as he named the rest of the team.* } \\
\text { gave explanations about the } \\
\text { session. }\end{array}$
\end{tabular}

P7: The virtual spatial environment, including lighting, sound or music, and visual effects, influences the attitudes of collaborators.

\begin{tabular}{lll}
\hline $\begin{array}{l}\text { The session took place } \\
\text { in a space simulating a }\end{array}$ & $\begin{array}{l}\text { After being named, each avatar } \\
\text { went to the place previously }\end{array}$ & $\begin{array}{l}\text { The trainees identified, per } \\
\text { the theoretical content they }\end{array}$ \\
partial area of an air- & defined in the theoretical con- had studied in Moodle, the \\
field: a main apron & tent (crew chief to the front of actions they should take, as \\
containing aircrafts, a & $\begin{array}{l}\text { the tractor, tractor operator next well as the precautions to be } \\
\text { runway, and a taxiway } \\
\text { to the crew chief and observers taken to ensure safe aircraft } \\
\text { where the aircraft tow } \\
\text { took place. }\end{array}$ \\
& $\begin{array}{l}\text { near the wing tips of the air- towing.* } \\
\text { craft). } \\
\text { The aircraft was towed from } \\
\text { point "A" to point "B".* }\end{array}$ \\
\end{tabular}

\section{Conclusions}

Cruz et al. provided the analysis of several cases that supported the propositions but some of their propositions had just a few chains of evidence or even a single chain. The point of having chains of evidences is to see if the related reference to a unit of analysis causes the behaviors stated by the proposition. If so, the proposition can be considered as true, or else it might be considered false. With our new data we found chains to support all propositions, some which reproduce Cruz et al.'s findings, and some which are entirely new, increasing the support to the validity of those propositions. All chains could be assigned to the propositions stated, meaning there is no need to created new propositions to fit the new data.

The existence of new chains makes us suspect that new cases might yield further chains. Hence, we deem it worthwhile to analyze more cases in search of chains of evidences that may lead us to create new propositions, or to the reformulation of the current ones, with possible impact in the answers to the research questions. Only 
when the analysis of more cases does not bring new chains of evidences, will we be confident that the set of propositions is consolidated.

This work started from the problem of lack of empirical data characterized by Cruz et al. [4] and applied the protocol created by these authors to a new case study. A simulator was developed, using OpenSim and a collaborative activity developed in an aeronautical maintenance scenario: the towing of an aircraft by a maintenance team. This simulator allowed, in a simple and low cost way, to apply the protocol and use this study the approach employed in the pilot case carried out by Cruz et al., i.e., using the same process in an unexplored topic. This drew our attention towards an aspect that wasn't considered earlier: many of its chains of evidence included gestures that were part of the learning content itself, e.g. "the gesture corresponding to the tractor moving forward". We wonder if Cruz et al.'s approach of broadly looking at all nonverbal elements shouldn't take into consideration two different aspects: non-verbal elements that are related to the learning content and non-verbal elements that not related to the learning content (e.g., spatial positioning identifying groupings or assemblies of participants). The implications of this are not clear and warrant further reflection and research towards a better understanding and framing of virtual worlds within the CSCW theoretical framework.

\section{References}

[1] Dreher, C., Reiners, T., Dreher, N., \& Dreher, H. (2009). 3D Virtual Worlds Enriching Innovation and Collaboration in Information Systems Research, Development, and Commercialisation. In Proceedings of the 2009 3rd IEEE International Conference on Digital Ecosystems and Technologies (pp. 168-173). Istambul, Turquia: IEEE

[2] Gul, L. F. (2008). Affording Embodiment in Collaborative Virtual Environments: Whats is the Role of Presence in Collaborative Design? In PRESENCE 2008: Proceendings of the 11th Annual International Workshop in Presence. Pádua, Itália: ISPR.

[3] Cruz, A., Paredes, H., Fonseca, B., Martins, P., \& Morgado, L. (2015). Collaboration in 3D Virtual Worlds: designing a protocol for case study research. In Workshop Proceedings of the 11th International Conference on Intelligent Environments (pp. 361372). IOS Press BV.

[4] Cruz, A., Paredes, H., Fonseca, B., Martins, P., Morgado, L. (2016). Collaboration in 3D Virtual Worlds: a protocol for case study research, EAI Endorsed Transactions on Future Intelligent Educational Environments, 2 (6), 151521-151521.

[5] Cruz, A., Paredes, H., Fonseca, B., Morgado, L., \& Martins, P. (2014). Can presence improve collaboration in 3D virtual worlds? In SLACTIONS 2013: Research conference on virtual worlds - Learning with simulations (Vol. 13, pp. 47-55). Vila Real, Portugal: Elsevier Procedia Technology

[6] De Nobrega, K. M., \& Rutkowski, A. F. (2012). Fostering Group Collaboration In Virtual Worlds. In Proceedings of the 2012 45th Hawaii International Conference on System Sciences (HICSS) (pp. 983-992). Maui, EUA: IEEE.

[7] Fominykh, M., Prasolova-Førland, E., \& Leong, P. (2012). Formal and Informal Collaborative Learning in 3D Virtual Campuses. In Proceedings of the Sixth 
International Conference on Collaboration Technologies (pp. 64-69). Barcelona, Espanha.

[8] Bente, G., \& Krämer, N. (2002). Virtual gestures : Analyzing social presence effects of computer-mediated and computer-generated nonverbal behaviour. In PRESENCE 2002: Proceedings of Fifth Annual International Workshop on Presence. Porto, Portugal: ISPR.

[9] Romano, D. M., Brna, P., \& Self, J. a. (1998). Collaborative decision-making and presence in shared dynamic virtual environments. In Proceedings of the Workshop on Presence in Shared Virtual Environments. BT Labs, Martlesham Heath. Ipswich, Reino Unido

[10] Bente, G., Rüggenberg, S., \& Krämer, N. (2004). Social Presence and Interpersonal Trust in Avatar-Based, Collaborative Net-Communications. In PRESENCE 2004: Proceedings of the 7th Annual International Workshop on Presence (pp. 54-61). Valência, Espanha: ISPR.

[11] Swinth, K. R., \& Blascovich, J. (2002). Perceiving and Responding to Others: HumanHuman and Human-Computer Social Interaction in Collaborative Virtual Environments. In PRESENCE 2002: 5th Annual International Workshop on Presence. Porto, Portugal: ISPR.

[12] Schmidt, K., \& Bannon, L. (1992). Taking CSCW Seriously: Supporting Articulation Work. Computer Supported Cooperative Work (CSCW): The Journal of Collaborative Computing and Work Pratices, 1, 7-40

[13] Cruz, A., Paredes, H., Fonseca, B., Martins, P., \& Morgado, L. (2015b). Fitting Three Dimensional Virtual Worlds into CSCW. In Proceedings of de 2015 IEEE 19th International Conference on Computer Supported Cooperative Work in Design (pp. 419-424). Calabria, Itália: IEEE.

[14] Yazan, B. (2015). Three Approaches to Case Study Methods in Education: Yin, Merriam, and Stake. The Qualitative Report, 20(2), 134-152.

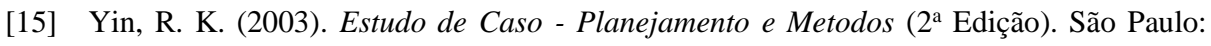
Bookman.

[16] Fonseca, B., Paredes, H., Rafael, L. J., Morgado, L., \& Martins, P. (2011). A Software Architecture for Collaborative Training in Virtual Worlds: F-16 Airplane Engine Maintenance. In Proceedings of the International Conference on Collaboration and Technology (pp. 102-109). Berlim Heidelberg, Alemanha: Springer-Verlag.

[17] Air Force AF/A4LM. (2015). Air Force Instruction 21-101 Aircraft and Equipment Maintenance Management. Disponível em http://www.e-publishing.af.mil/index.asp

[18] Pinheiro, A., Fernandes, P., Maia, A., Cruz, G., Pedrosa, D., Fonseca, B., ... Rafael, J. (2012). Development of a mechanical maintenance training simulator in OpenSimulator for F-16 aircraft engines. Procedia Computer Science, 15, 248-255. 